\title{
Formation of $\mathrm{NiMoO}_{4}$ Anisotropic Nanostructures under Hydrothermal Conditions
}

\author{
T. L. Simonenko ${ }^{a}$ *, V. A. Bocharova ${ }^{a, b}$, N. P. Simonenko ${ }^{a}$, E. P. Simonenko ${ }^{a}$, \\ V. G. Sevastyanov ${ }^{a}$, and N. T. Kuznetsov ${ }^{a}$ \\ ${ }^{a}$ Kurnakov Institute of General and Inorganic Chemistry, Russian Academy of Sciences, Moscow, 119991 Russia \\ ${ }^{b}$ Mendeleev University of Chemical Technology of Russia, Moscow, 125047 Russia \\ *e-mail: egorova.offver@gmail.com \\ Received July 9, 2021; revised August 2, 2021; accepted August 4, 2021
}

\begin{abstract}
The synthesis of NiMoO4 hierarchical nanostructures using the hydrothermal method has been studied. The decomposition of $\mathrm{NiMoO}_{4} \cdot x \mathrm{H}_{2} \mathrm{O}$ crystalline hydrate formed during the synthesis has been studied using synchronous thermal analysis upon heating in a stream of air and argon. According to X-ray diffraction as well as scanning and transmission electron microscopies, the proposed conditions allow one to synthesize single-phase nanosized (average CSR size of about $25 \pm 2 \mathrm{~nm}$ ) nickel(II) molybdate, which has a spinel-type monoclinic structure (space group $C 2 / \mathrm{m}$ ) without impurity inclusions.
\end{abstract}

Keywords: hydrothermal synthesis, nanopowder, hierarchical structures, anisotropic structures, nickel molybdate, spinel, NiMoO4, supercapacitor

DOI: $10.1134 / \mathrm{S} 0036023621120160$

\section{INTRODUCTION}

The implementation of efficient storage of electricity is one of the most important tasks in ensuring the sustainable development of modern society. In this regard, significant efforts of scientists and engineers are focused on the development of portable, energyintensive, environmentally friendly and at the same time inexpensive energy accumulation and storage devices [1-3]. One of the promising candidates in this context are electrochemical capacitors or supercapacitors, which demonstrate a high charge/discharge rate (on the order of several seconds), high cyclic stability, and specific power in a wide range of operating temperatures (from -50 to $+50^{\circ} \mathrm{C}$ ), as well as a higher operational safety and service life compared to lithium-ion batteries [4-6]. The listed advantages provide serious competitive advantages of supercapacitors in such areas as electric transport, development of portable (including flexible and wearable) electronics, creation of non-volatile memory systems, uninterruptible power supplies, etc. [7, 8]. According to the principle of energy storage, supercapacitors can be divided into two types: (i) supercapacitors with an electric double layer, on the surface of which charge accumulates during the adsorption/desorption of ions, and (ii) supercapacitors with a pseudocapacitive effect, in which energy is accumulated not only through the operation of an electric double layer but also due to reversible redox reactions occurring on the surface or in the near-surface region of the active layer of elec- trodes $[9,10]$. Devices of the second type, as a rule, are characterized by a higher specific capacity [11].

The performance of a supercapacitor is also largely determined by the electrode material. The most popular electrode materials for pseudocapacitive supercapacitors are transition metal oxides such as $\mathrm{MnO}_{2}$, $\mathrm{Co}_{3} \mathrm{O}_{4}, \mathrm{NiO}, \mathrm{Co}(\mathrm{OH})_{2}$, and $\mathrm{Ni}(\mathrm{OH})_{2}$ [12-14]. However, in recent years, there has been a growing interest in the study of electrode materials for supercapacitors based on metal molybdates, in particular, $\mathrm{NiMoO}_{4}$, $\mathrm{CoMoO}_{4}$, and $\mathrm{MnMoO}_{4}$, which are characterized by high electrical conductivity and redox activity [1518]. It should be noted that electrodes based on nickel molybdate make it possible to achieve the highest values of specific capacity among materials of the family of metal molybdates, which is due to the high electrochemical activity of the nickel ion in the composition of $\mathrm{NiMoO}_{4}[19,20]$. An additional improvement in the functional properties of the materials of the electrodes of supercapacitors can be achieved through the directed design of their microstructure. According to modern literature data, the production of nanomaterials (powders and coatings) in the form of hierarchical structures makes it possible, as a rule, to significantly increase the target characteristics of not only the final material but also the device being developed as a whole [21-24]. In particular, it was demonstrated that the production of an active electrode material in the form of nanorods [25-27], nanospheres [28-30], nanofibers [31-33], nanosheets [34-36], and nanoflowers 
[37-39] allows one achieving high values of specific capacity and rate of charge/discharge, as well as increase the service life of the supercapacitor. Analysis of the literature data showed that one of the most popular and convenient methods for producing nanomaterials for creating alternative energy devices in the form of both powders and coatings with a hierarchical organization is the hydrothermal method [17, 40-42]. In this work, as a preliminary stage of synthesis (formation of the embryonic phase), the programmed coprecipitation of nickel and molybdenum hydroxides was carried out; the thus obtained disperse system was further subjected to hydrothermal treatment. The method of programmed coprecipitation, in comparison with the classical technique, makes it possible to carry out an automated synthesis process with a high degree of reproducibility, and also makes it possible to vary the parameters of the deposition process over a wider range $[43,44]$.

The aim of this work is to study the formation of 1D nickel molybdate nanostructures under hydrothermal conditions, as well as to study the thermal behavior, crystal structure, and morphology of the resulting powder.

\section{EXPERIMENTAL}

Nanopowder of composition $\mathrm{NiMoO}_{4}$ was obtained in accordance with the following scheme. A weighed portion of urea $(0.362 \mathrm{~g})$ was added with constant stirring to an aqueous solution of salts $\mathrm{NiCl}_{2} \cdot 6 \mathrm{H}_{2} \mathrm{O}$ and $\left(\mathrm{NH}_{4}\right)_{6} \mathrm{Mo}_{7} \mathrm{O}_{24} \cdot 4 \mathrm{H}_{2} \mathrm{O}(0.2 \mathrm{~mol} / \mathrm{L})$. The reaction mixture was transferred into a steel autoclave with a Teflon insert and subjected to hydrothermal treatment at a temperature of $120^{\circ} \mathrm{C}$ for $2 \mathrm{~h}$. Then, the system was naturally allowed to cool to $25^{\circ} \mathrm{C}$. The separation and washing of the formed dispersed phase with distilled water was carried out by cyclic centrifugation, after which it was dried at $100^{\circ} \mathrm{C}$ for $3 \mathrm{~h}$. Then, the obtained powder was heat treated $\left(500^{\circ} \mathrm{C}, 2 \mathrm{~h}\right)$ in oxidizing and inert atmospheres under conditions of simultaneous thermal analysis.

Synchronous (TGA/DSC) thermal analysis of the powder obtained during hydrothermal synthesis and subsequent drying at $100^{\circ} \mathrm{C}$ was carried out using an SDT Q-600 thermal analyzer (controlled heating was carried out in $\mathrm{Al}_{2} \mathrm{O}_{3}$ microcrucibles in the temperature range $25-500^{\circ} \mathrm{C}$ at a rate of $10 \mathrm{~K} / \mathrm{min}$ with further exposure at $500^{\circ} \mathrm{C}$ for $2 \mathrm{~h}$ in an argon flow and an air flow, the gas flow rate was $250 \mathrm{~mL} / \mathrm{min}$, the weight of the sample was $27.3080 \mathrm{mg}$ ).

In order to record the IR transmission spectra of the powders, we prepared the corresponding suspensions in Nujol mull, which were then placed in the form of a film between glasses of potassium bromide. Spectral analysis was performed in the wavenumber range of $350-4000 \mathrm{~cm}^{-1}$ (signal accumulation time was $15 \mathrm{~s}$, and the resolution was $1 \mathrm{~cm}^{-1}$ ) using an Infralum FT-08 IR Fourier spectrometer.

$\mathrm{X}$-ray powder diffraction studies of the obtained powders were performed using a Bruker D8 Advance diffractometer $\left(\mathrm{Cu} K_{\alpha}\right.$ radiation, $\lambda=1.5418 \AA$, Ni filter, $E=40 \mathrm{keV}, I=40 \mathrm{~mA}, 2 \theta=5^{\circ}-80^{\circ}$, resolution $0.02^{\circ}$, signal accumulation time at point $0.3 \mathrm{~s}$ ).

The morphology and elemental composition of the formed oxide powder was studied using scanning electron microscopy on a Carl Zeiss Nvision 40 threebeam workstation equipped with an Oxford Instruments X-MAX 80 energy dispersive microprobe analyzer. The size and shape of the obtained oxide particles were studied by transmission electron microscopy on a Jeol JEM-1011 microscope equipped with an Orius SC1000W digital camera.

\section{RESULTS AND DISCUSSION}

According to the data of synchronous (TGA/DSC) thermal analysis of the powder after drying at $100^{\circ} \mathrm{C}$, its behavior in an air flow and in an argon flow is generally similar. Thus, when heated to $500^{\circ} \mathrm{C}$, there are three main stages of weight loss: in the ranges $25-190$ (4.7\%), $190-415(5.1 \%)$, and $415-500^{\circ} \mathrm{C}(2 \%)$ (Fig. 1). The first stage (accompanied by a weak endothermic effect) is associated with the removal of the residual solvent, the second stage (the endothermic effect of medium intensity with a minimum at $409^{\circ} \mathrm{C}$ ) is associated with the irreversible process of removal of structurally bound water molecules and the phase transition of $\mathrm{NiMoO}_{4} \cdot x \mathrm{H}_{2} \mathrm{O}$ hydrate into oxide, and the third stage, with gradual transformation of the crystal structure of intermediate products and the formation of $\alpha-\mathrm{NiMoO}_{4}$. At the first stage of exposure at $500^{\circ} \mathrm{C}$, an insignificant increase in the powder mass was observed, followed by a decrease. As a result, the final weight loss during heat treatment was $11.8 \%$. Thus, based on the data obtained in the course of thermal analysis, the optimal mode of heat treatment $\left(500^{\circ} \mathrm{C}\right.$, $2 \mathrm{~h}$, argon or air) of the powder was determined in order to crystallize the target oxide.

The IR spectra (Fig. 2, spectra 2 and 3) of powders heat-treated at $500^{\circ} \mathrm{C}$ in a flow of air and in an argon flow show a double absorption band with maxima at 963 and $935 \mathrm{~cm}^{-1}$, as well as bands with maxima at 597 and $450 \mathrm{~cm}^{-1}$, which indicate the formation of a spinel with the $\alpha-\mathrm{NiMoO}_{4}$ structure. At the same time, the absorption bands in the range $920-830 \mathrm{~cm}^{-1}$ observed after drying the powder at $100^{\circ} \mathrm{C}$ (Fig. 2, spectrum 1) indicate the presence of molybdenum oxide powder [21]. In addition, the powder after drying is characterized by the presence of a more intense absorption band in the region of $3000-3700 \mathrm{~cm}^{-1}$ (stretching vibrations of the $\mathrm{OH}$ groups) and an absorption band with a maximum at $1620 \mathrm{~cm}^{-1}$ (bending vibrations of the $\mathrm{OH}$ groups) related to the molecules of absorbed water in the structure of the powder, which also indirectly con- 


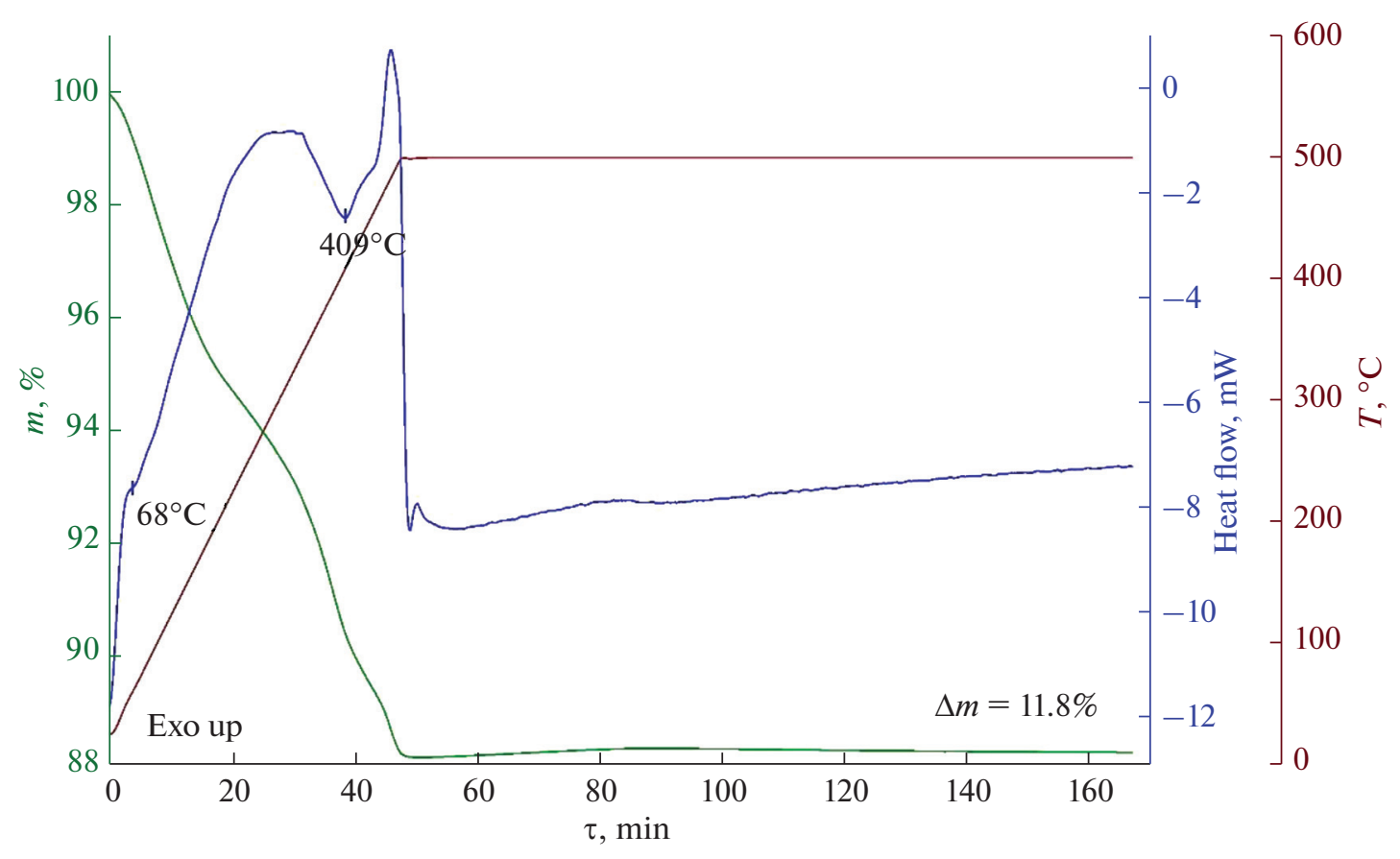

Fig. 1. TGA (green) and DSC (blue) curves of products of hydrothermal synthesis of $\mathrm{NiMoO}_{4}$ in an argon flow; the red curve represents the heating mode.

firms the formation of hydrate $\mathrm{NiMoO}_{4} \cdot x \mathrm{H}_{2} \mathrm{O}$ under these conditions. It should be noted that the spectra of nanopowders $\mathrm{NiMoO}_{4}$ obtained upon heat treatment in argon and air are similar, which indicates that the presence of an inert atmosphere is not a prerequisite for the formation of the target structure of $\alpha-\mathrm{NiMoO}_{4}$.

According to the $\mathrm{X}$-ray powder diffraction results (Fig. 3), under the conditions of hydrothermal synthesis followed by drying at $100^{\circ} \mathrm{C}$, a crystalline hydrate of the composition $\mathrm{NiMoO}_{4} \cdot x \mathrm{H}_{2} \mathrm{O}$ is formed, which agrees with the results of thermal analysis and IR spectroscopy, and further heat treatment at $500^{\circ} \mathrm{C}$ leads to the formation of single-phase target nickel(II) molybdate with a monoclinic spinel structure (space group $C 2 / m$, JCPDS No. 33-0948) [21]. The average CSR size $(25 \pm 2 \mathrm{~nm})$ of the powder after additional heat treatment at $500^{\circ} \mathrm{C}$ was calculated using the Scherrer formula: $d=K \lambda / \beta \cos \theta$, where $d$ is the average CSR size, $K=0.9$ in the approximation that the powder particles have a spherical shape, $\lambda$ is the $X$-ray radiation wavelength, $\beta$ is the reflection width at half maximum, and $\theta$ is the diffraction angle. As can be seen from the X-ray powder diffraction pattern, the obtained nanopowder oxide does not contain crystalline impurities of the initial reagents, as well as any intermediate or by-products.

The microstructure of the powder obtained during heat treatment at $500^{\circ} \mathrm{C}$ was studied using scanning (Fig. 4) and transmission (Fig. 5) electron microscopies. It was determined that the substance has a uniform morphology and consists of organized anisotro- pic particles, which are layered one-dimensional nanostructures with an average diameter of $\sim 80 \mathrm{~nm}$ (the ratio of length to diameter is $\sim 16$ ), consisting of nanorods with a diameter of $\sim 10 \mathrm{~nm}$ (the ratio of length to diameter is $\sim 130$ ). Probably, the agglomeration of individual nanorods and the formation of layered 1D structures with two levels of particle organization occurs even at the stage of hydrothermal treatment, preventing of which requires the introduction of

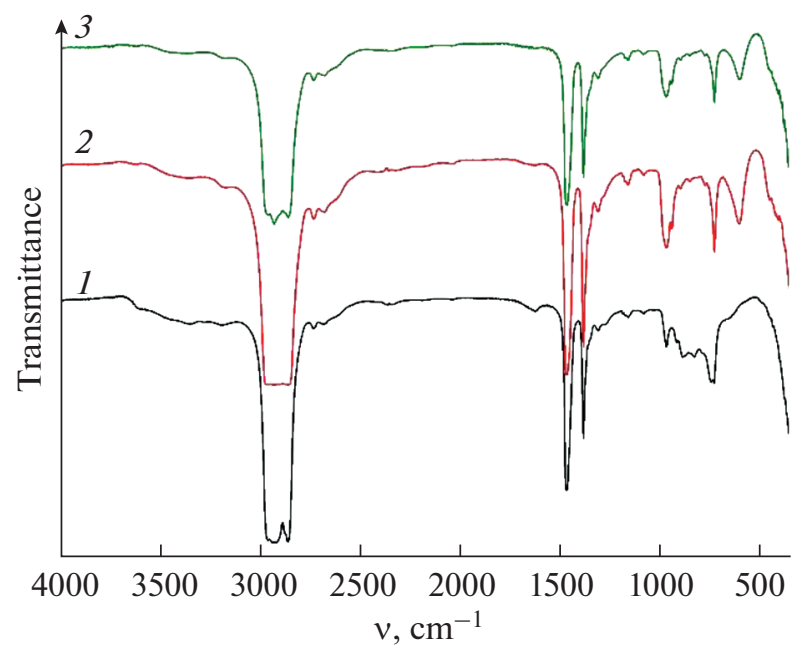

Fig. 2. IR spectra of $\mathrm{NiMoO}_{4}$ nanopowders obtained by (1) hydrothermal synthesis after drying at $100^{\circ} \mathrm{C}$ and $(2,3)$ subsequent heat treatment at $500^{\circ} \mathrm{C}(2$ in an argon flow; 3 in an air flow). 


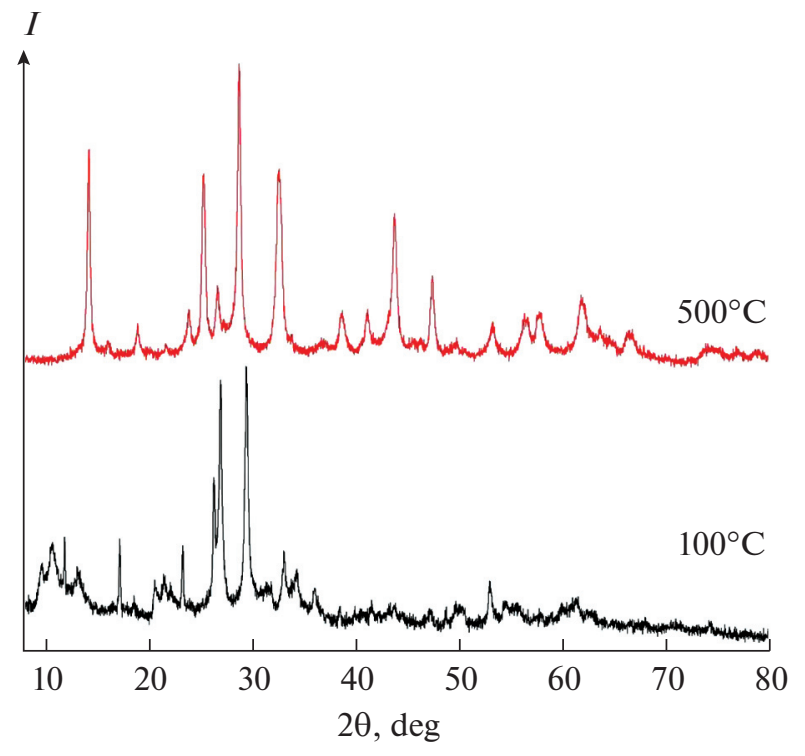

Fig. 3. X-ray diffraction pattern of $\mathrm{NiMoO}_{4}$ powder obtained after heat treatment in an argon atmosphere at $500^{\circ} \mathrm{C}$.

appropriate surfactants into the reaction system. The results of microscopy also indicate the absence of impurities that differ in particle shape from the bulk of the substance, while the study of the material in the contrast mode by the average atomic number (reflected electron detector) and the results of the energy dispersive elemental microanalysis confirm the formation of homogeneous nickel(II) molybdate.

In the course of the study, it was shown that the hydrothermal method is an effective method for the formation of layered anisotropic $\mathrm{NiMoO}_{4}$ nanostructures with a monoclinic spinel structure, consisting of nanorods $\sim 10 \mathrm{~nm}$ in diameter, and their developed surface makes this approach promising for creating supercapacitor electrodes with a pseudocapacitive effect.

\section{CONCLUSIONS}

The process of formation of layered anisotropic nanostructures $\mathrm{NiMoO}_{4}$ with a monoclinic spinel structure under hydrothermal conditions has been studied. According to the data of IR spectroscopy, $\mathrm{X}$-ray powder diffraction, and thermal analysis, under the conditions of hydrothermal synthesis followed by drying at $100^{\circ} \mathrm{C}$, a crystalline hydrate of the composition $\mathrm{NiMoO}_{4} \cdot x \mathrm{H}_{2} \mathrm{O}$ is formed, and further heat treatment at $500^{\circ} \mathrm{C}$ in a flow of air or argon leads to the formation of single-phase nickel molybdate(II) with a monoclinic spinel structure (space group $C 2 / \mathrm{m}$ ) and an average CSR size of about $25 \pm 2 \mathrm{~nm}$.

Using scanning and transmission electron microscopies, it was shown that the resulting compound has a homogeneous morphology and consists of organized anisotropic particles, which are layered 1D nanostructures with an average diameter of about $80 \mathrm{~nm}$ (the ratio of length to diameter is $\sim 16$ ), consisting of nanorods with a diameter of about $10 \mathrm{~nm}$ (ratio of length to diameter is $\sim 130$ ). The study of the material in the contrast mode by the average atomic number (reflected electron detector) and the results of the energy dispersive elemental microanalysis confirm the formation of homogeneous nickel(II) molybdate.

Thus, the proposed approach can be recommended for the synthesis of hierarchical nanostructures of the $\mathrm{NiMoO}_{4}$ composition with a developed surface in the creation of functional oxide nanomaterials, including for use in alternative energetics, in particular, in the manufacture of supercapacitor electrodes with a pseudocapacitive effect.
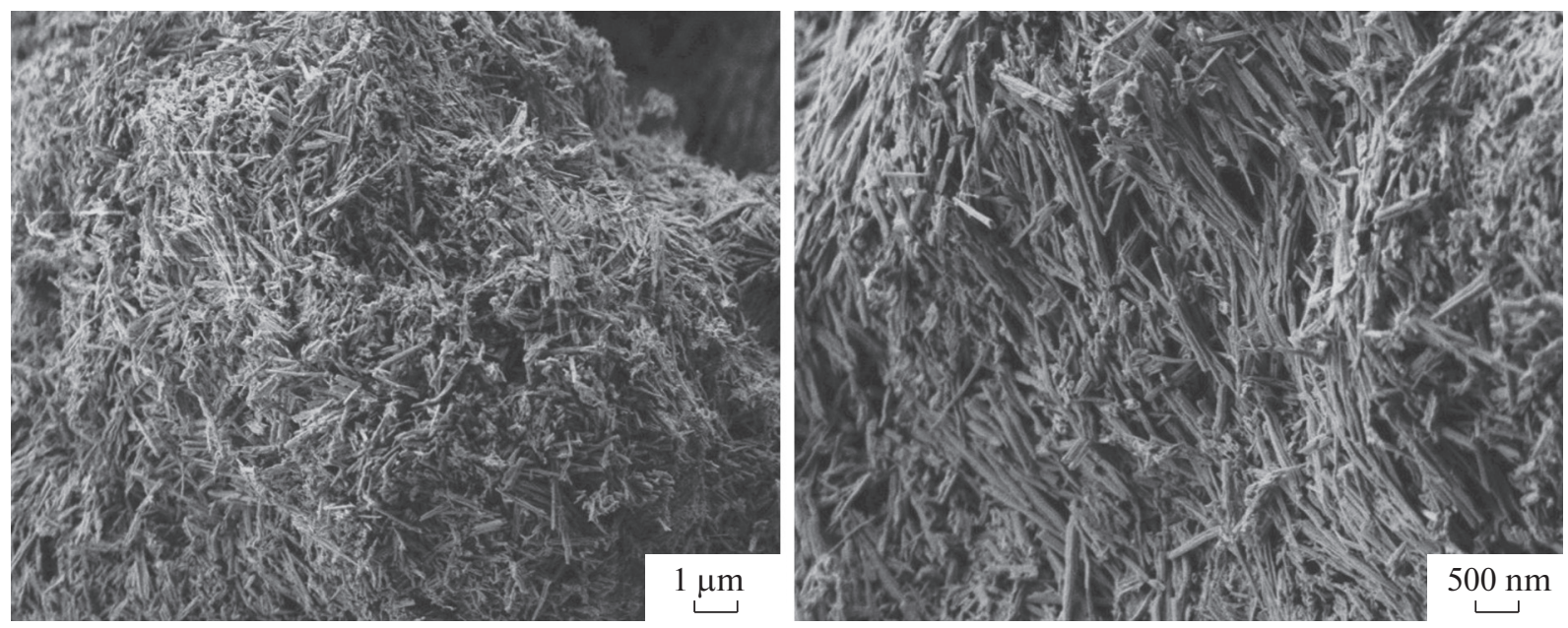

Fig. 4. Microstructure of $\mathrm{NiMoO}_{4}$ powder after heat treatment in argon at $500^{\circ} \mathrm{C}$ (according to SEM data). 

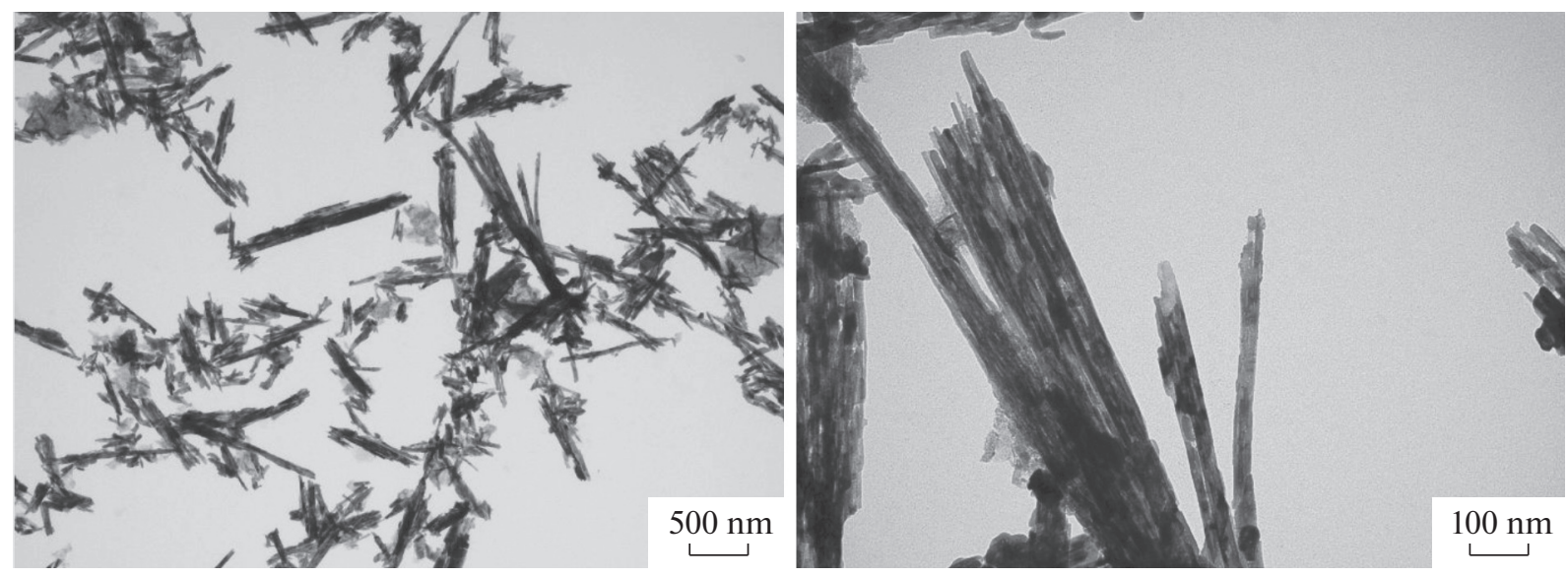

Fig. 5. Microstructure of $\mathrm{NiMoO}_{4}$ powder after heat treatment in argon at $500^{\circ} \mathrm{C}$ (according to TEM data).

\section{ACKNOWLEDGMENTS}

X-ray powder diffraction and SEM studies were carried out using shared experimental facilities supported by IGIC RAS state assignment.

\section{FUNDING}

This work was partially supported by a Scholarship of the President of the Russian Federation for young scientists and graduate students (project SP-2407.2019.1, synthesis of nanostructures of the NiMoO4 nanopowders) and the Ministry of Science and Higher Education of the Russian Federation within the framework of the State Assignment of the Kurnakov Institute of General and Inorganic Chemistry, Russian Academy of Sciences (X-ray diffraction patterns and SEM studies of the obtained nanopowder).

\section{CONFLICT OF INTEREST}

The authors declare that they have no conflicts of interest.

\section{OPEN ACCESS}

This article is licensed under a Creative Commons Attribution 4.0 International License, which permits use, sharing, adaptation, distribution and reproduction in any medium or format, as long as you give appropriate credit to the original author(s) and the source, provide a link to the Creative Commons license, and indicate if changes were made. The images or other third party material in this article are included in the article's Creative Commons license, unless indicated otherwise in a credit line to the material. If material is not included in the article's Creative Commons license and your intended use is not permitted by statutory regulation or exceeds the permitted use, you will need to obtain permission directly from the copyright holder. To view a copy of this license, visit http://creativecommons.org/licenses/by/4.0/.

\section{REFERENCES}

1. A. González, E. Goikolea, J. A. Barrena, et al., Renew. Sustain. Energy Rev. 58, 1189 (2016). https://doi.org/10.1016/j.rser.2015.12.249

2. M. Salanne, B. Rotenberg, K. Naoi, et al., Nat. Energy 1, 16070 (2016). https://doi.org/10.1038/nenergy.2016.70

3. Y. Shao, M. F. El-Kady, J. Sun, et al., Chem. Rev. 118, $9233(2018)$.

https://doi.org/10.1021/acs.chemrev.8b00252

4. S. Rahimi, S. Shahrokhian, and H. Hosseini, J. Electroanal. Chem. 810, 78 (2018). https://doi.org/10.1016/j.jelechem.2018.01.004

5. C. An, Y. Zhang, H. Guo, et al., Nanoscale Adv. 1, 4644 (2019).

https://doi.org/10.1039/c9na00543a

6. C. Lu and X. Chen, Acc. Chem. Res. 53, 1468 (2020). https://doi.org/10.1021/acs.accounts.0c00205

7. Y. Wang, Y. Song, and Y. Xia, Chem. Soc. Rev. 45, 5925 (2016). https://doi.org/10.1039/C5CS00580A

8. L. Li, Z. Lou, D. Chen, et al., Small 14, 1702829 (2018). https://doi.org/10.1002/smll.201702829

9. Y. Wang, Y. Song, and Y. Xia, Chem. Soc. Rev. 45, 5925 (2016). https://doi.org/10.1039/C5CS00580A

10. G. Wang, L. Zhang, and J. Zhang, Chem. Soc. Rev. 41, 797 (2012). https://doi.org/10.1039/C1CS15060J

11. M. Salanne, B. Rotenberg, K. Naoi, et al., Nat. Energy 1, 16070 (2016). https://doi.org/10.1038/nenergy.2016.70

12. J. Jiang, Y. Li, J. Liu, et al., Adv. Mater. 24, 5166 (2012). https://doi.org/10.1002/adma.201202146

13. J. Yan, Q. Wang, T. Wei, et al., Adv. Energy Mater. 4, 1300816 (2014).

14. T. L. Simonenko, N. P. Simonenko, P. Y. Gorobtsov, et al., J. Alloys Compd. 832, 154957 (2020).

https://doi.org/10.1016/j.jallcom.2020.154957 
15. T. L. Simonenko, V. A. Bocharova, N. P. Simonenko, et al., Russ. J. Inorg. Chem. 66, 1633 (2021). https://doi.org/10.1134/S0036023621110176

16. G. K. Veerasubramani, K. Krishnamoorthy, S. Radhakrishnan, et al., Int. J. Hydrogen Energy 39, 5186 (2014). https://doi.org/10.1016/j.ijhydene.2014.01.069

17. D. Cheng, Y. Yang, J. Xie, et al., J. Mater. Chem. A 3, 14348 (2015). https://doi.org/10.1039/c5ta03455h

18. D. Cai, D. Wang, B. Liu, et al., ACS Appl. Mater. Interfaces 5, 12905 (2013). https://doi.org/10.1021/am403444v

19. D. Muthu, S. Vargheese, Y. Haldorai, et al., Mater. Sci. Semicond. Process. 135, 106078 (2021). https://doi.org/10.1016/j.mssp.2021.106078

20. D. Guo, P. Zhang, H. Zhang, et al., J. Mater. Chem. A 1, 9024 (2013). https://doi.org/10.1039/c3ta11487b

21. C. Mazzocchia, C. Aboumrad, C. Diagne, et al., Catal. Lett. 10, 181 (1991). https://doi.org/10.1007/BF00772070

22. V. Augustyn, P. Simon, and B. Dunn, Energy Environ. Sci. 7, 1597 (2014) https://doi.org/10.1039/c3ee44164d

23. A. Burke, J. Power Sources 91, 37 (2000). https://doi.org/10.1016/S0378-7753(00)00485-7

24. C. Cui, J. Xu, L. Wang, et al., ACS Appl. Mater. Interfaces 8, 8568 (2016). https://doi.org/10.1021/acsami.6b02962

25. R. Yang, X. Guo, K. Song, et al., Ceram. Int. 47, 11349 (2021). https://doi.org/10.1016/j.ceramint.2020.12.261

26. E. R. Ezeigwe, P. S. Khiew, C. W. Siong, et al., Ceram. Int. 43, 13772 (2017). https://doi.org/10.1016/j.ceramint.2017.07.092

27. S. K. Ray, D. Dhakal, J. K. Sohng, et al., Chem. Eng. J. 347, 366 (2018). https://doi.org/10.1016/j.cej.2018.04.122

28. Z. Zhang, Y. Liu, Z. Huang, et al., Phys. Chem. Chem. Phys. 17, 20795 (2015). https://doi.org/10.1039/C5CP03331D

29. D. Cai, D. Wang, B. Liu, et al., ACS Appl. Mater. Interfaces 5, 12905 (2013). https://doi.org/10.1021/am403444v
30. M. Yao, Z. Hu, Y. Liu, et al., Ionics (Kiel) 22, 701 (2016). https://doi.org/10.1007/s11581-015-1587-8

31. L. Zhou, S. Zeng, D. Zheng, et al., Chem. Eng. J. 400, 125832 (2020). https://doi.org/10.1016/j.cej.2020.125832

32. S. Deshagani and D. Maity, A. Das, et al., ACS Appl. Mater. Interfaces 13, 34518 (2021). https://doi.org/10.1021/acsami.1c07064

33. Y. Zhao, Y. Liu, J. Du, et al., Appl. Surf. Sci. 487, 442 (2019). https://doi.org/10.1016/j.apsusc.2019.05.142

34. H. Xuan, R. Wang, J. Yang, et al., J. Mater. Sci. 56, 9419 (2021). https://doi.org/10.1007/s10853-021-05902-5

35. Q. Hu, C. Kang, S. Cao, et al., J. Alloys Compd. 883, 160867 (2021). https://doi.org/10.1016/j.jallcom.2021.160867

36. Y. Meng, D. Yu, Y. Teng, et al., J. Energy Storage 29, 101195 (2020). https://doi.org/10.1016/j.est.2020.101195

37. B. Ramulu, S. Chandra Sekhar, G. Nagaraju, et al., Appl. Surf. Sci. 515, 146023 (2020). https://doi.org/10.1016/j.apsusc.2020.146023

38. C. J. Raj, R. Manikandan, K. H. Yu, et al., Inorg. Chem. Front. 7, 369 (2020). https://doi.org/10.1039/C9QI01085H

39. A. K. Yedluri, T. Anitha, and H. J. Kim, Energies 12, 1143 (2019).

40. T. L. Simonenko, V. A. Bocharova, P. Y. Gorobtsov, et al., Russ. J. Inorg. Chem. 65, 1304 (2020). https://doi.org/10.1134/S003602362009018

41. T. L. Simonenko, V. A. Bocharova, P. Y. Gorobtsov, et al., Russ. J. Inorg. Chem. 65, 1292 (2020). https://doi.org/10.1134/S0036023620090193

42. Z. Yu, L. Tetard, L. Zhai, et al., Energy Environ. Sci. 8, 702 (2015). https://doi.org/10.1039/c4ee03229b

43. T. L. Simonenko, N. P. Simonenko, P. Yu, et al., J. Colloid Interface Sci. 588, 209 (2021). https://doi.org/10.1016/j.jcis.2020.12.052

44. T. L. Simonenko, N. P. Simonenko, E. P. Simonenko, et al., Russ. J. Inorg. Chem. 64, 1475 (2019). https://doi.org/10.1134/S0036023619120167

Translated by $V$. Avdeeva 